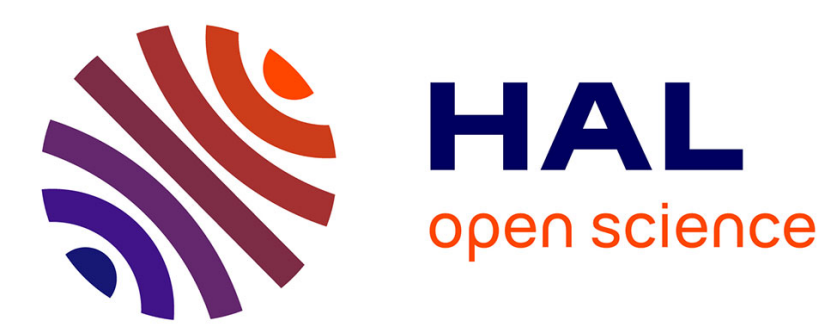

\title{
Are beliefs a matter of taste? A case for Objective Imprecise Information
}

Raphaël Giraud, Jean-Marc Tallon

\section{To cite this version:}

Raphaël Giraud, Jean-Marc Tallon. Are beliefs a matter of taste? A case for Objective Imprecise Information. Theory and Decision, 2011, 71 (1), pp.23-32. 10.1007/s11238-010-9197-4 . halshs00502781

\section{HAL Id: halshs-00502781 https://shs.hal.science/halshs-00502781}

Submitted on 15 Jul 2010

HAL is a multi-disciplinary open access archive for the deposit and dissemination of scientific research documents, whether they are published or not. The documents may come from teaching and research institutions in France or abroad, or from public or private research centers.
L'archive ouverte pluridisciplinaire $\mathbf{H A L}$, est destinée au dépôt et à la diffusion de documents scientifiques de niveau recherche, publiés ou non, émanant des établissements d'enseignement et de recherche français ou étrangers, des laboratoires publics ou privés. 


\title{
Are Beliefs a Matter of Taste? A case for Objective Imprecise Information
}

\author{
Raphaël Giraud \\ CRESE \\ University of Franche-Comté*
}

\author{
Jean-Marc Tallon \\ Paris School of Economics \\ Université Paris I Panthéon-Sorbonne ${ }^{\dagger}$
}

\begin{abstract}
We argue, in the spirit of some of Jean-Yves Jaffray's work, that explicitly incorporating the information, however imprecise, available to the decision maker is relevant, feasible and fruitful. In particular, we show that it can lead us to know whether the decision maker has wrong beliefs and whether it matters or not, that it makes it possible to better model and analyze how the decision maker takes into account new information, even when this information is not an event and finally that it is crucial when attempting to identify and measure the decision maker's attitude toward imprecise information.
\end{abstract}

Journal of Economic Literature: B41,D80.

\section{Introduction}

When facing a decision under uncertainty, gathering information about the odds of various events relevant to the outcome of the decision seems a good idea and indeed is common practice. Jean-Yves Jaffray, trained as an engineer, also thought so. Indeed, he made a distinction between complete ignorance, when no information is available and situations in which some information is available, although not sufficient to pin down uniquely a probability distribution; situations he labeled "imprecise risk". This did not fit in the traditional distinction between risk (covered by von Neumann and Morgenstern) and uncertainty (covered by Savage). Risk is too specific a situation to be widely used, while Savage's treatment of uncertainty (following the subjectivist tradition of de Finetti) potentially misses some important data available to the modeler. ${ }^{1}$

\footnotetext{
*Corresponding author: raphael.giraud@univ-fcomte.fr

${ }^{\dagger}$ Centre d'Economie de la Sorbonne, 106 Bd de l'Hopital. Jean-Marc.Tallon@univ-paris1.fr

${ }^{1}$ What is at stake is not so much the expected utility aspect of Savage's theory (although we will also take issue with this) but the fact that Savage's approach does not give any tool to model available information.
} 
Jean-Yves Jaffray always advocated that decision theorists should develop pragmatic tools to use all data available, in the most "objective way", thus distancing himself from a purely behavioral approach. This entails some cost as one sometimes needs to take as granted the information available to the decision maker. But it also entails gains, in terms of aid to decision making. It is also surprisingly very much in line with common practice in economics, where situations of risk are commonly assumed (e.g. industrial organization and incentive theory, in macroeconomics, labor economics...), assuming, often implicitly, that enough information is available to form these probabilistic beliefs. Jean-Yves Jaffray however wanted to also consider situations where information does not come as a probability distribution. This stance thus begs the question "how to handle available information, when it is not probabilistic?” Jean-Yves Jaffray in his 1989 piece considered objects (belief functions) that generalize usual probabilistic tools. He had to work hard to do so (see Wakker's piece in this issue) but the result is remarkably elegant.

In this short piece, would like to argue that this general approach to decision making is 1) relevant, 2) feasible and 3) fruitful. We will almost exclusively focus the discussion on static problems.

\section{Why a theory incorporating available informa- tion?}

Why should one need a theory incorporating explicitly how available information affects the decision maker's choice? We already have vNM, which covers risky situations and hasn't Savage proved that, in the end, decision making under uncertainty can be reduced to the same expected utility criterion, only with subjective beliefs?

We can see two problems with this coherent but somewhat simplistic view of decision theory under uncertainty. The first is that subjective beliefs can be quite arbitrary and hence the jump from saying that Savage and vNM criteria look alike to, say, assuming the same subjective beliefs for all agents in a model is unwarranted. The second is that even in relatively simple situations when some information is available (e.g. Ellsberg) a decision maker may fail to come up with probabilistic subjective beliefs and hence Savage's nice construction might not apply in some (most?) cases. Let's take these two points in turn.

Let's start with a somewhat provocative statement. There is, we claim, a kind of folk theorem in economic theory saying that one could explain anything if one were to allow arbitrary beliefs. And indeed from the rational expectations revolution to various game theoretical equilibrium concepts, a lot of research has been dedicated to make sure beliefs could not just be anything. But this concerns beliefs on endogenous variables (to make it short, prices or strategies). When it comes to beliefs on 
exogenous events, economic theory often makes the assumption of common probabilistic beliefs among the agents. It rarely justifies this assumption, or when it does, invokes some principle according to which different beliefs would lead to infinite speculative trade, or in a different version, argue that decision makers with the same information should hold the same beliefs. A statement that cannot be expressed using Savage's framework (and is known to be flawed even in a dynamic setting with arrival of new, common, information -see Diaconis \& Freedman (1986); Feldman (1991).)

If two agents hold different probabilistic beliefs on some event, it has to be the case that one is mistaken if one assumes an underlying "true" process, and economic theory is not necessarily keen on deriving results on assumptions like that (although less taboo nowadays given the strength of the behavioral approach). This might be a rationale for actually assuming in a lot of models that agents all have the same probabilistic beliefs, de facto reducing the situation to a situation of risk. Hence, we argue, a good part of economic theory rests on this (very often implicit) assumption that the setting under consideration is one of risk. This would thus justify our claim that part of economic theory is based on the assumption that there is, in the background, some probabilistic information available to all decision makers. Following this claim, one could then wonder why this approach has (almost) not been generalized to non probabilistic information. A possible answer goes back to the construction of Savage, which gives a rationale for not bothering with say, sets of probabilities.

This brings us to our second point, raised above, which is of a different nature. It criticizes the idea that under uncertainty, one can always come up with probabilistic beliefs, be they subjective. Thus, the reduction to a setting of risk is not warranted. However, failure to come up with probabilistic beliefs does not mean that there is no structure on the beliefs of the agents.

So, there is room for a decision theory that would incorporate as a primitive the information agents have (in probabilistic or not probabilistic ways) which generalizes decision under risk and does not necessarily reduce to it. Next, we argue that there are ways to deal with available information even though it does not come as a probability distribution, i.e., that the tools for such a theory already exist and can already be used to study economic issues.

\section{Imprecise risk and imprecise information}

The idea of generalizing the notion of risk, i.e. known probability distribution, to some notion of imprecise risk, i.e., imprecisely known probability distribution, dates back to Jaffray (1989), who proposed to replace lotteries by belief functions, that roughly speaking correspond to sets of lotteries. He obtains a representation in the 
spirit of the Arrow-Hurwicz criterion (Arrow \& Hurwicz, 1972). This idea was recently revived by several authors who proposed to model imprecise risk either by necessity measures (Rébillé, 2006) or directly by sets of lotteries (Stinchcombe, 2003; Olszewski, 2007; Ahn, 2008).

The above approach consists in assuming imprecise information on the distribution of outcomes, and preferences over such imprecise lotteries. A different approach, pioneered by Wang (2001), is to consider that the DM has imprecise information on the set of events, and preferences on pairs $(f, \mathscr{P})$ where $f$ is an act and $\mathscr{P}$ is a set of probability distributions over the state space. This approach reduces to the previous one only if one assumes that if two such pairs generate the same set of lotteries, they are indifferent to the DM, so that the source of uncertainty does not matter. Wang actually takes as primitives triples $\left(f, \mathscr{P}, P^{*}\right)$ where $P^{*}$ is a reference prior. Wang's main result is to provide axiomatic foundations for a general version of the minimum relative entropy principle of Anderson et al. (1999). He also characterizes a maxmin rule whereby the decision maker maximizes the minimum expected utility with respect to the set of objective priors. This rule is characterized by an axiom of aversion to uncertainty à la Gilboa-Schmeidler (1989), which, in his framework takes the form of an axiom of strong aversion to objective ambiguity. It says that the decision maker should always prefer an act in a situation characterized by a set of prior to the same act in a situation characaterized by a set of prior that would be a superset of the former. In the same setting, Gajdos et al. (2004) weaken the notion of aversion to objective ambiguity, that they call aversion to imprecision, and obtain a generalized maxmin rule: the decision maker maximizes the minimum expected utility over a subset $\varphi(\mathscr{P})$ of the set of initial priors. One special case of this model is a form of perturbed expected utility, given by the formula

$$
V\left(f, \mathscr{P}, P^{*}\right)=(1-\varepsilon) \int u \circ f \mathrm{~d} P^{*}+\varepsilon \min _{P \in \mathscr{P}} \int u \circ f \mathrm{~d} P,
$$

a model that was first proposed by Ellsberg in order to rationalize the behavior described in his seminal paper. It also appears in Tapking (2004) in the context of the study of updating rules for capacities. Gajdos et al. (2008) generalizes Gajdos et al. (2004) by dispensing with the reference prior, now part of the representation. Kopylov (2006) provides alternative foundations for the perturbed expected utility formula, characterizing it by an axiom that can be interpreted as a combination of ambiguity aversion and loss aversion. In the same setting, Giraud (2009) provides an axiomatization for nonadditive second order beliefs over $\mathscr{P}$, that serve for the aggregation of the various expected utilities of an act $f$ under information $\mathscr{P}$. The analogous of $\varphi(\mathscr{P})$ is here a nonadditive probability $\rho^{\mathscr{P}}$ on the state space.

Nehring $(2007,2009)$ studies a related framework where, along with the usual preference relation, the decision maker is endowed with a (potentially incomplete) 
comparative likelihood relation, that is assumed to represent the decision maker's beliefs, but that could just as well be interpreted it as available information, betting preferences being interpreted as revealed beliefs. The object of investigation is the compatibility of betting preferences as derived from the preference relation with the decision maker's beliefs represented by the comparative likelihood relation.

Finally, Gilboa et al. (Forth.a) also distinguish two preference relations, one of which, potentially incomplete, is interpreted as preferences based on "hard information", and the other, a completion of the former, is revealed by actual choices. They provide an axiomatic derivation of a maxmin expected utility representation for the revealed preferences relation with respect to a set of priors that represents the "objective" preference relation.

\section{Fruitfulness of the approach}

Taking into account available information allows one to deal with three important issues that cannot be addressed otherwise. First, incorporating available information can lead us to know whether the decision maker has wrong beliefs and how this does or does not matter. Second, incorporating available information makes it possible to better model and analyze how the decision maker takes into account new information, even when this information is not an event. Thirdly, it is crucial when identifying and measuring the decision maker's attitude toward imprecise information.

\subsection{Are Rational Beliefs Reasonable?}

Savage's project, when writing the Foundations of Statistics was, among other things, to provide axioms for a rational behavior in the presence of uncertainty. He often identified rationality with a form of consistency, as exemplified by axioms P2 and P4. If we accept the definition of rationality as consistency, and if by beliefs we mean beliefs revealed by the decision maker's willingness to bet, then beliefs satisfying P2 and $\mathrm{P} 4$ must be rational.

However, there are other ways of defining the rationality of beliefs. One is to ask whether they contradict some information available at least to the observer. If they don't, we might call them rational, or, at least, reasonable. An example of unreasonable beliefs occurs if the decision maker is willing to bet on the fact that I am the king of France, while abiding by Savage's principles. Sen's concept of "rational fool", though used in a different context, seems to apply, since believing things that are not true is a step in the direction of insanity. The point is here that in Savage's framework it is not possible to distinguish between rational fools and people in their right mind, whether rational in Savage's sense or not. ${ }^{2}$ For that, we

\footnotetext{
${ }^{2}$ This point has been made in various places. See for a recent and forceful formulation the work of Itzhak
} 
need an explicit formalization of the information available to the decision maker.

Things are actually a little more complicated than that, since we have to distinguish different kind of unreasonable beliefs:

- Beliefs that contradict information that is available to the modeler but not to the DM.

- Beliefs that contradict information known by the modeler to be available to the DM.

In the first case, it is difficult to say whether the DM is being reasonable or not by holding these beliefs: it would be to some extent unfair to qualify these beliefs as unreasonable, even though they are known by the modeler to be wrong. Consider for example a tribe that has lived for centuries away from western civilization and holds some beliefs about biology (e.g. that ingesting sperm will help a boy to become a man, the rationale of many initiation rites) that to a certain extent make sense given its state of knowledge even though they are wrong.

The second case, on the other hand, does not seem ambiguous: it is really unreasonable to known something to be true, and not to act accordingly (i.e., in Savage's framework, to prefer betting on events known to have low probability rather than on events known to have high probability). This kind of behavior has received a lot of attention in the philosophical literature since Aristotle, under the name of the "weakness of the will" problem: I know what is good for me, yet I don't do it. In the decision-theoretic literature, this problem is often studied in the context of preferences over menus, with beliefs not playing a decisive role. But there is an obvious connection between this problem and the contradiction between available information and beliefs revealed by choices. This is implicit in the analysis of this problem in terms of sequential decision: consider the problem of starting an addictive behavior. I might do it, deceiving myself into believing that I will be able to stop when I want to, even though there is plenty of information around telling that I will not. It seems that a fully rational DM will know that, and never start.

There are however arguments that suggests that things might not be that clear after all. These arguments, brought forth in papers such as Akerlof \& Dickens (1982), Brunnermeier \& Parker (2005) and Gollier (2005), go along the following line: it might be optimal to deceive oneself in order to feel better overall, even though that might increase the risk of experiencing damages. For instance, I may choose to underestimate the probability to be contaminated by the HIV in order to better enjoy sex, even though that may lead me to engage in more dangerous sexual practices. To the extent that these wrong beliefs, or illusions, are chosen to optimize some

Gilboa and coauthors in a series of papers (Gilboa et al., 2008, 2009, Forth.b; Gilboa, 2009). Our focus here is however slightly different, since we want to emphasize that there is a way out of this, while in these papers the focus is more on the philosophical question of the relevance of Bayesianism per se. 
overall well-being criterion, they are not irrational from the point of view of standard economic theory, although they might be deemed unreasonable.

One last important issue here is the question of how much does the DM trust the information available to him. Distrust would be revealed by choices as some discrepancy between information and behavior, and may be considered rational, if the DM has good reasons to distrust the information, e.g. when it comes from experts known to be unreliable. But this means that the DM has private information, and begs the question: how is the observer/modeler to treat private information?

All this boils down to the deeper question of the status of what is sometimes called "objective" information. One possible definition of objective information is one over which there is no possible disagreement. Information that not everyone trusts doesn't count as objective, therefore, as long as the distrust is based on reliable evidence. If we adopt a weaker definition of "objective" as meaning: beliefs that are not derived from the DM's observed behavior, then obviously this allows for some discrepancy between objective information and revealed beliefs that is not necessarily irrational or unreasonable. Therefore it is essential, for the interpretation of decision models, to have a perfectly clear interpretation of the primitive that model available information.

\subsection{Attitude Toward New Information}

Modeling available information has also some advantage when dealing with arrival of new information. This information may or may not be describable as an event of the state space. For instance consider the case where ambiguity stems from the unsettled debate between experts on a scientific issue, say the appropriateness of a large scale vaccination against influenza A. Assume that each expert comes up with a probability distribution over side-effects, and the DM is the government that must make a decision about whether to start the vaccination campaign or not. Consider now two pieces of information. The first is that one side-effect that was deemed possible by some and not by others actually occurs in a randomized experiment. This is an event. The second is that one of the experts dies (or, less tragically, that two experts decide to form an association and to submit only one estimate). This is not an event. However it is obviously an ambiguity reducing information, and should matter to the DM.

If new information is not describable as an event, then in the purely subjectivist theory there is no way to model how the DM deals with it. On the other hand, if we model available information as a set of probability distributions, and if new information comes as a new set of distributions, the revision of beliefs becomes a simple matter. It suffices to replace the previous information set $\mathscr{P}$ by the new one $\mathscr{Q}$, and proceed to apply any decision criterion that represents preferences over act- 
information pairs.

\subsection{Measure of imprecision aversion}

Various models have been proposed to deal with situations where information is too scarce to pin down a single probability distribution. One prominent model is Gilboa \& Schmeidler (1989)'s Maxmin Expected Utility. The interpretation of the set of priors in this model is not obvious, however. If the revealed set of priors coincides, so to speak, with the actual objective information, taking decisions on the basis of the smallest expected utility is an extremely pessimistic, perhaps unreasonable, attitude, as noticed in Wang (2003) and discussed in section 2. But, if the revealed set of priors is actually much smaller than the maximal one compatible with the available information, then taking the minimum expected utility with respect to this smaller set is not that pessimistic after all. There is no way one can settle this without knowing what exactly this set of priors is, and in the context of fully subjective axiomatizations, we simply cannot do it for lack of a sufficiently rich setup. It must be noted, incidentally, that Ghirardato et al. (2004)'s axiomatization of a generalization of Gilboa \& Schmeidler (1989)'s model designed to differentiate ambiguity and ambiguity attitude and hence immune to the accusation of excessive pessimism allows only (as the authors make perfectly clear) to differentiate between revealed ambiguity and attitude toward revealed ambiguity, since it is cast in exactly the same setup as Gilboa \& Schmeidler (1989)'s, and cannot therefore completely succeed in settling down the controversy.

This interpretational problem translates into a measurement problem if one does not incorporate available information. And indeed, in experimental work, one usually uses the available information to try to capture or measure ambiguity attitude (see e.g., Halevy (2007) or Ahn et al. (2008)).

In Jean-Yves Jaffray's 1989 model of imprecise risk, such an interpretational issue does not arise. Since the set of priors coincide by construction with the available information, the coefficient weighting the minimum expected utility is a coefficient of pessimism. In a similar vein, in Gajdos et al. (2008), introducing explicitly the information available to the decision maker makes it possible to define concepts related to attitude toward imprecision, such as comparative aversion to bet imprecision, and to show that a decision maker $a$ is more averse to bet imprecision than $b$ if and only if $\varphi^{b}(\mathscr{P}) \subseteq \varphi^{a}(\mathscr{P})$. Moreover, in the so-called "contraction model" of equation (1) with endogenous $P^{*}$, it is shown that $a$ is more averse to bet imprecision than $b$ if and only if $\varepsilon^{a} \geq \varepsilon^{b}$. $\varepsilon$ is also shown to be interpretable as a "relative imprecision aversion premium". Similarly, in Giraud (2009) it is shown that $a$ is more averse to bet imprecision than $b$ if and only if $\rho_{b}^{\mathscr{P}} \geq \rho_{b}^{\mathscr{P}}$, i.e. if $b$ always has a higher willingness to bet than $a$, indicating a form of optimism. 


\section{References}

Ahn, David, Choi, Syngjoo, Gale, Douglas, \& Kariv, Shachar. 2008. Estimating Ambiguity Aversion in a Portfolio Choice Experiment. Tech. rept. UC Berkeley.

Ahn, David S. 2008. Ambiguity Without a State-Space. Review of Economic Studies, 75(1), 3-28.

Akerlof, G.A., \& Dickens, W.T. 1982. The economic consequences of cognitive dissonance. The American Economic Review, 72(3), 307-319.

Anderson, E., Hansen, L., \& Sargent, T. 1999. Robustness, Detection and the Price of Risk. University of Chicago.

Arrow, Kenneth, \& Hurwicz, Leonard. 1972. An optimality criterion for decisionmaking under ignorance. In: Carter, C. F., \& Ford, J.L. (eds), Uncertainty and Expectations in Economics. Oxford, England: Basil Blackwell \& Mott Ltd.

Brunnermeier, M.K., \& Parker, J.A. 2005. Optimal expectations. The American Economic Review, 95(4), 1092-1118.

Diaconis, P., \& Freedman, D. 1986. On the consistency of Bayes estimates. The Annals of Statistics, 14(1), 1-26.

Ellsberg, D. 1961. Risk, ambiguity, and the Savage axioms. Quartely Journal of Economics, 75, 643-669.

Feldman, M. 1991. On the generic nonconvergence of Bayesian actions and beliefs. Economic Theory, 1(4), 301-321.

Gajdos, Thibault, Tallon, Jean-Marc, \& Vergnaud, Jean-Christophe. 2004. Decision making with imprecise probabilistic information. Journal of Mathematical Economics, 40(6), 647-681.

Gajdos, Thibault, Hayashi, Takashi, Tallon, Jean-Marc, \& Vergnaud, JeanChristophe. 2008. Attitude toward Imprecise Information. Journal of Economic Theory, 140(1), 23-56.

Ghirardato, Paolo, Maccheroni, Fabio, \& Marinacci, Massimo. 2004. Differentiating Ambiguity and Ambiguity Attitude. Journal of Economic Theory, 118(2), 133173.

Gilboa, I., \& Schmeidler, D. 1989. Maxmin expected utility with a non-unique prior. Journal of Mathematical Economics, 18, 141-153.

Gilboa, I., Postlewaite, A.W., \& Schmeidler, D. 2008. Probability and Uncertainty in Economic Modeling. Journal of Economic Perspectives, 22(3), 173-188.

Gilboa, I., Postlewaite, A., \& Schmeidler, D. 2009. Is it always rational to satisfy Savage's axioms? Economics and Philosophy, forthcoming.

Gilboa, I., Maccheroni, F., Marinacci, M., \& Schmeidler, D. Forth.a. Objective and Subjective rationality in a multiple prior model. Econometrica. 
Gilboa, I., Postlewaite, A., \& Schmeidler, D. Forth.b. Rationality of Belief. Or Why Bayesianism is Neither Necessary Nor Sufficient for Rationality. Synthese.

Gilboa, Itzhak. 2009. Questions in Decision Theory. Annual Review in Economics.

Giraud, Raphaël. 2009. Objective Imprecise Probabilistic Information, Second Order Beliefs and Ambiguity Aversion: an Axiomatization. mimeo.

Gollier, C. 2005. Optimal Illusions and Decisions under Risk. IDEI Working Papers.

Halevy, Y. 2007. Ellsberg Revisited: An Experimental Study. Econometrica, 75(2), 503-536.

Jaffray, Jean-Yves. 1989. Généralisation du critère de l'utilité à l'incertain régulier. Operations Research/ Recherche Opérationnelle, 23(3), 237-267.

Kopylov, Igor. 2006. A Parametric Model of Ambiguity Hedging. University of California at Irvine.

Nehring, Klaus. 2007 (April). Bernouilli Without Bayes: A Theory of UtilitySophistiicated Preferences under Ambiguity. Mimeo, University of California, Davis.

Nehring, Klaus. 2009. Imprecise Probabilistic Beliefs as a Context for DecisionMaking under Ambiguity. Journal of Economic Theory. Mimeo, University of California, Davis.

Olszewski, Wojciech. 2007. Preferences Over Sets of Lotteries. Review of Economic Studies, 74(04), 567-595.

Rébillé, Yann. 2006. Decision making over necessity measures through the Choquet integral criterion. Fuzzy Sets and Systems, 157(23), 3025-3039.

Stinchcombe, M. 2003. Choice and games with ambiguity as sets of probabilities. mimeo,University of Texas, Austin.

Tapking, Jens. 2004. Axioms for preferences revealing subjective uncertainty and uncertainty aversion. Journal of Mathematical Economics, 40, 771-797.

Wang, Tan. 2001, revised 2003. A Class of Multi-Priors Preferences. University of British Columbia. 